\title{
Microvascular Decompression for Glossopharyngeal Neuralgia in the Semi-Sitting Position: A Report of Two Cases
}

\author{
Shinjitsu Nishimura, ${ }^{1}$ Keiichi Kubota, ${ }^{1}$ Sumito Okuyama, ${ }^{1}$ Junko Matsuyama, ${ }^{1}$ \\ Ken Kazama, ${ }^{1}$ Masato Tomii, ${ }^{1}$ Tadao Matsushima, ${ }^{1}$ Masato Kurihara ${ }^{2}$ and \\ Kazuo Watanabe ${ }^{1}$
}

${ }^{1}$ Department of Neurosurgery, Southern Tohoku General Hospital, Iwanuma, Miyagi, Japan

${ }^{2}$ Department of Anesthesiology, Southern Tohoku General Hospital, Iwanuma, Miyagi, Japan

\begin{abstract}
The semi-sitting position is well known to neurosurgeons. However, there are few reports of microvascular decompression surgery for glossopharyngeal neuralgia performed using the semi-sitting position. The semi-sitting position is not widely adopted in Japan, but it is considered to be a very useful neurosurgical position. Microvascular decompression surgery for glossopharyngeal neuralgia is a relatively rare procedure, and the semi-sitting position is very effective, considering the possibility of intraoperative cardiac arrest and postoperative complications of lower cranial nerve palsy. This report describes two cases of glossopharyngeal neuralgia operated in the semi-sitting position. Microvascular decompression was performed on both patients, and postoperative pain controls were good and no complications were observed. We show that the use of the semi-sitting position to perform microvascular decompression for glossopharyngeal neuralgia provides an excellent surgical view of the brainstem.
\end{abstract}

Keywords: functional neurosurgery; glossopharyngeal neuralgia; half-sitting position; microvascular decompression; semi-sitting position

Tohoku J. Exp. Med., 2021 July, 254 (3), 183-188.

\section{Introduction}

Glossopharyngeal neuralgia is less common than trigeminal neuralgia and is rarely experienced in clinical practice. The diagnosis is difficult, and the related surgical procedures require special attention. Specifically, intraoperative stimulation of the vagus nerve causes vital changes such as bradycardia and hypotension. In addition, there is a risk of postoperative lower cranial nerve palsy.

Since April 2017, our institute has utilized the semisitting position in surgery for posterior fossa lesions in patients aged $<60$ years with no evidence of cardiac shunt on preoperative echocardiography. The semi-sitting position is well known to neurosurgeons. However, there are few reports of microvascular decompression in the semisitting position for glossopharyngeal neuralgia. We report two such cases here to highlight the effectiveness and safety of the semi-sitting position in decompression surgery.

\section{Case Presentation}

Case 1

A 59-year-old woman presented with an approximately 15-month history of left-sided sore throat, mild swallowing difficulties, and hoarseness. She was admitted to our department and showed no improvement with oral medications. The source image of three-dimensional timeof-flight magnetic resonance angiography (3D TOF MRA) and its maximum intensity projection (MIP) rendering revealed arteries in contact with the left-sided lower cranial nerves in a loop (Fig. 1A, B). Furthermore, the left vertebral artery (VA) trunk was slightly tortuous, suggesting a cause for the symptoms of glossopharyngeal neuralgia. After induction of general anesthesia, a central venous catheter was placed through the right subclavian vein under ultrasound guidance to remove air bubbles from the right atrium. A precordial Doppler (HiDop 300, Medizintechnik Basler AG, Zurich, Switzerland) attached to the patient had been used to detect a venous air embolism during surgery.

Received April 8, 2021; revised and accepted May 18, 2021. Published online July 15, 2021; doi: 10.1620/tjem.254.183.

Correspondence: Shinjitsu Nishimura, M.D., Department of Neurosurgery, Southern Tohoku General Hospital, 1-2-5 Satonomori, Iwanuma, Miyagi 989-2483, Japan.

e-mail: snishimura@minamitohoku.jp

(C)2021 Tohoku University Medical Press. This is an open-access article distributed under the terms of the Creative Commons Attribution-NonCommercial-NoDerivatives 4.0 International License (CC-BY-NC-ND 4.0). Anyone may download, reuse, copy, reprint, or distribute the article without modifications or adaptations for non-profit purposes if they cite the original authors and source properly.

https://creativecommons.org/licenses/by-nc-nd/4.0/ 

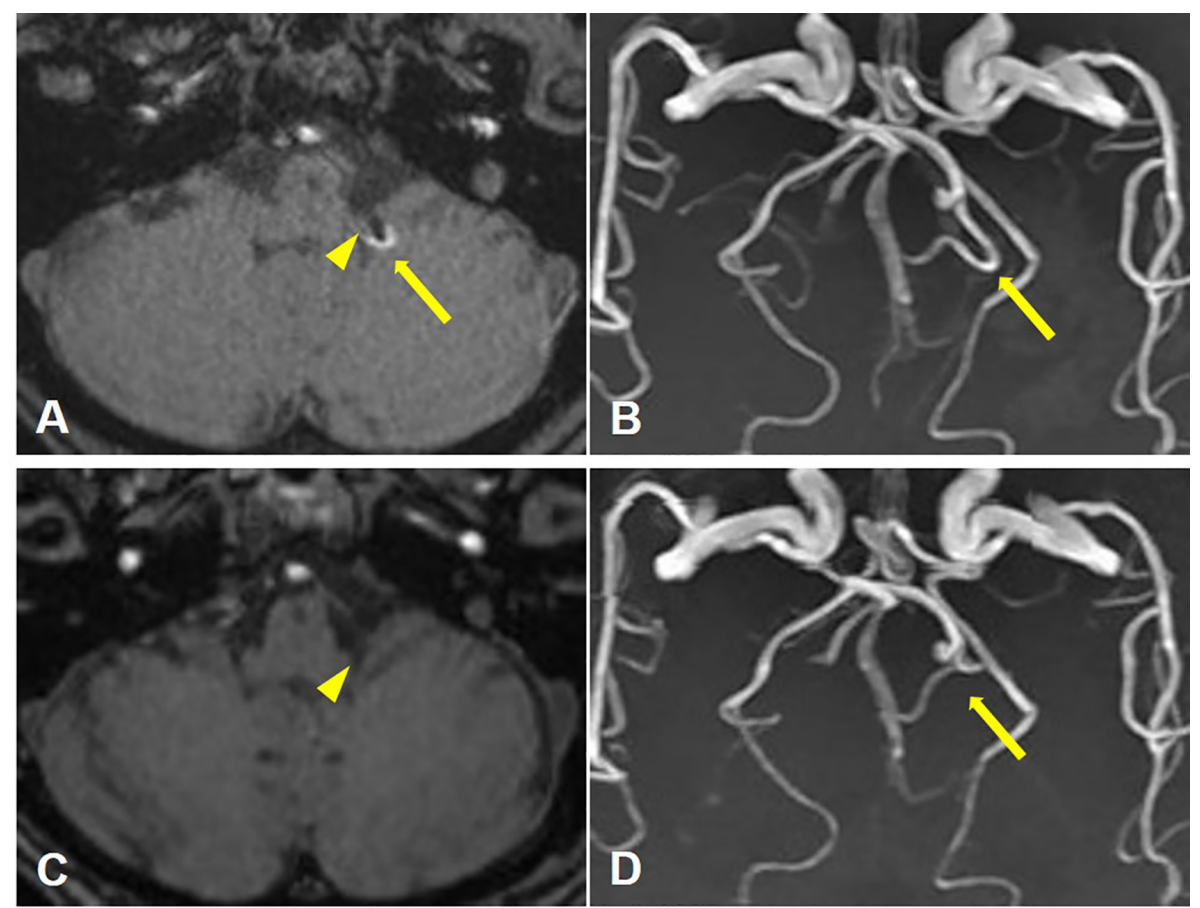

Fig. 1. Pre- and post-operative magnetic resonance images of Case 1.

A. Pre-operative axial source image of three-dimensional time-of-flight magnetic resonance angiography (3D TOF MRA). The left posterior inferior cerebellar artery (PICA) is in contact with the root entry zone (REZ) of the glossopharyngeal nerve. The arrowhead indicates a REZ and the arrow indicates a PICA loop. B. Pre-operative axial maximum intensity projection (MIP) rendering. The arrow indicates a PICA loop. C. Post-operative axial source image of 3D TOF MRA. Neurovascular contacts around the REZ of the glossopharyngeal nerve were adequately released. The arrowhead indicates a REZ. D. Post-operative axial maximum intensity projection (MIP) rendering. The arrow shows the release of the PICA loop.

The back plate of the operating table was raised by approximately $30^{\circ}$ and the leg plate was raised by approximately $45^{\circ}$. Furthermore, a special polyurethane mattress was inserted underneath the patient's back, curving and raising the back to achieve the semi-sitting position. Both upper limbs were raised to be almost parallel to the ground at shoulder level (Fig. 2A).

A three-pin head clamp and crossbar adapter (QR3 Headrest System; DORO, Freiburg, Germany) was used. The patient's head was flexed ventrally, rotated approximately $30^{\circ}$ ipsilaterally, and tilted slightly to the side contralateral to the one planned for surgical approach. When measured, the bed-up angle of the operating table backplate was about $27^{\circ}$ with respect to the horizontal plane.

We performed a small craniotomy in the same location as that of a microvascular decompression (MVD) for hemifacial spasm (HFS). An approximately 6-cm straight skin incision was followed by a circular craniotomy approximately $2.5 \mathrm{~cm}$ in diameter, located below the inferior nuchal line, just behind the mastoid process (Fig. 2B).

After opening the dura, the cerebellum was gently elevated using a retractor to expose the surgical field. Interference with the root entry zone (REZ) of the left glossopharyngeal nerve was indirectly related to the VA trunk and directly to the posterior inferior cerebellar artery (PICA) loop.
The microscope was positioned to look upward from below, to observe the REZ and to move the VA trunk. After transferring the VA trunk to the pyramidal side with pieces of a small fibrinogen sealant patch (TachoSil ${ }^{\circledR}$ Patch), the PICA loop was pulled away from the REZ and transpositioned toward the pyramidal side and the cerebellar hemisphere in a direction away from the REZ, using Teflon tape with fibrin glue (Fig. 2C-F). No air embolism (AE) was detected during the operation.

On postoperative magnetic resonance imaging (MRI), the PICA loop was adequately displaced away from the REZ (Fig. 1C, D). The patient's symptoms began improving from day 5 postoperatively, and symptoms fully resolved on postoperative day 20. There were no new symptoms of lower cranial nerve dysfunction. No recurrence was observed within 30 months of follow-up.

\section{Case 2}

A 41-year-old woman presented with an approximately 18 -month history of sore throat affecting the left side. Oral treatment was administered, but she experienced repeated exacerbations separated by periods of remission, and pain control was becoming less attainable over time. As in Case 1, the PICA loop appeared to be the offending vessel, but the VA trunk was not involved (Fig. 3A, B). Surgery was performed in the same manner as described for 

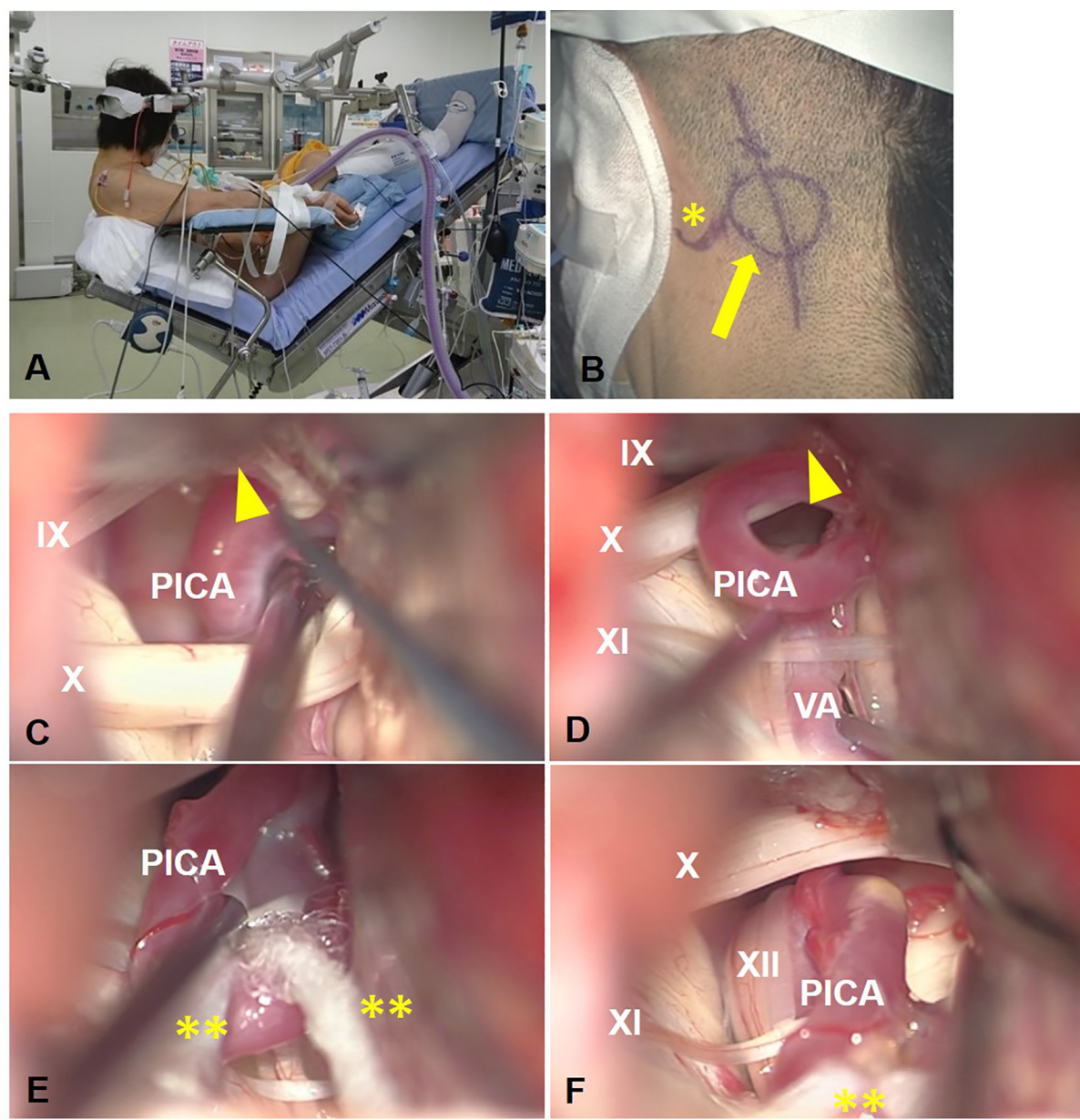

Fig. 2. Surgical procedure performed in Case 1.

A. Lateral view of the patient in the semi-sitting position. The back plate of the operating table was raised by approximately $30^{\circ}$ and, simultaneously, the leg plate was raised by approximately $45^{\circ}$. Furthermore, a special polyurethane mattress then inserted under the patient's back, curving the back and bringing it forward. B. The skin incision and craniotomy marking. The asterisk indicates the mastoid process, and the arrow indicates the microscope angle. C. Intraoperative images of the left-sided microvascular decompression (MVD) of the glossopharyngeal nerve. Confirmation of the posterior inferior cerebellar artery (PICA) loop between the glossopharyngeal (IX) and vagus (X) nerves. The arrowhead indicates the root entry zone (REZ). D. A small piece of TachoSil ${ }^{\circ}$ was inserted between the vertebral artery (VA) trunk and the pyramidal bone following fixation of the VA trunk laterally. Then, the PICA loop is pulled out to the caudal side of the vagus (X) nerve. The arrowhead indicates the REZ. E. The PICA loop is pulled up toward the pyramidal side and the cerebellar hemisphere with Teflon tape. F. The PICA loop moved to the caudal aspect of the glossopharyngeal and vagus $(\mathrm{X})$ nerves.

IX, glossopharyngeal nerve; X, vagus nerve; XI, spinal accessory nerve; XII, hypoglossal nerve; VA, vertebral artery; PICA, posterior inferior cerebellar artery.

*Mastoid process, **Teflon tape.

Case 1. The only responsible vessel was the PICA loop (Fig. 4A, B). Transposition of the PICA loop away from the REZ was performed using Teflon tape (Fig. 4C, D). The bed-up angle of the operating table back-plate was approximately $30^{\circ}$. There was no AE during the surgery.

On the postoperative MRI, the PICA loop was fully decompressed from the REZ (Fig. 3C, D). The patient's pain improved immediately postoperatively and did not recur during the 27-month follow-up. Furthermore, no complications or lower cranial nerve symptoms were observed.

Informed consent was obtained from both patients, and this manuscript was approved by the local institutional review board.

\section{Discussion}

MVD for glossopharyngeal neuralgia was first proposed by Jannetta (Laha and Jannetta 1977) and is now an established treatment. The PICA is the most common blood vessel responsible for glossopharyngeal neuralgia secondary to neurovascular compression (NVC). Three types of NVC have been described: Type I, NVC at the glossopharyngeal nerve REZ within the retro-olivary sulcus; Type II, the shoulder of the VA causes NVC at the glossopharyngeal 

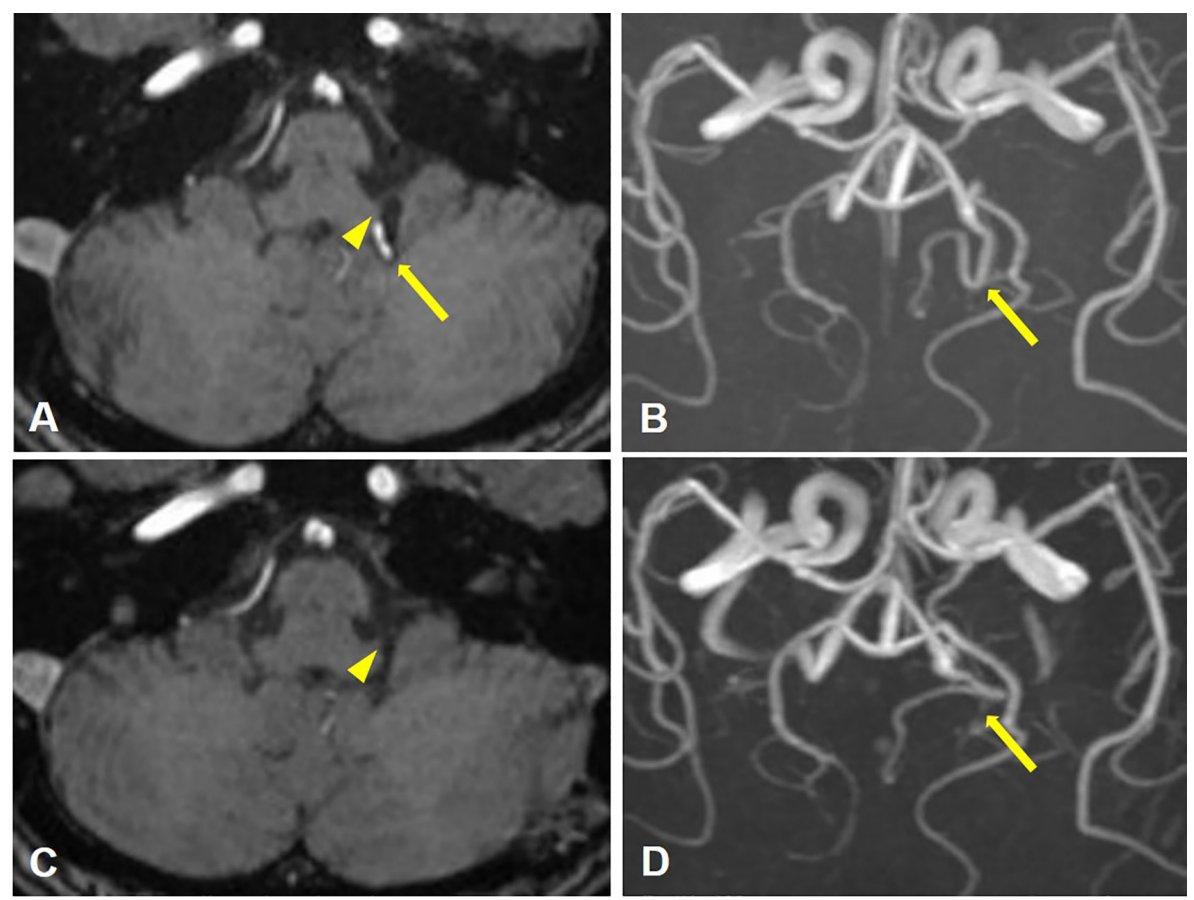

Fig. 3. Pre- and post-operative magnetic resonance images of Case 2.

A. Pre-operative axial source image of three-dimensional time-of-flight magnetic resonance angiography (3D TOF MRA). The arrowhead indicates a root entry zone (REZ) and arrow indicates a posterior inferior cerebellar artery (PICA) loop. B. Pre-operative axial maximum intensity projection (MIP) rendering. The arrow indicates a PICA loop. C. Post-operative axial source image of 3D TOF MRA. The arrowhead indicates a REZ. PICA loop around the REZ was disappeared. D. Post-operative axial MIP rendering. The arrow indicates the transpositioned PICA.

nerve REZ; and Type III, a "sandwich-like" compression in which the VA and the PICA in combination cause NVC (Tanrikulu et al. 2015). The offending vessels were the VA trunk and PICA loop (Type III) in Case 1 and the PICA loop alone in Case 2 (Type I).

Our surgical procedures were performed through small craniotomies in the same location as those performed for MVD in the treatment of HFS. Although they were similar to those of the HFS REZ, in this procedure it was necessary to look upward along the side of the brainstem from below for a clear view (Kawashima et al. 2010; RevueltaGutierrez et al. 2016). In both cases, the semi-sitting position allowed a view from below and ensured adequate decompression (in Case 1, for observing the REZ, managing the VA trunk, and transpositioning the PICA loop; in Case 2, to observe the REZ and ensure the transpositioning of the PICA loop).

Surgery in the semi-sitting position is particularly useful in posterior fossa surgery (Samii and Matthies 1996, 1997; Malis 1998). The semi-sitting position reduces the risk of $\mathrm{AE}$ by not lowering the legs. In our department, the upper body is raised up to approximately $30^{\circ}$; further, by raising the lower limbs, we reduce the risk of AE. We confirmed that the angle of head elevation that caused clinically significant $\mathrm{AE}$ was $35.7^{\circ}$ in our previous study (Kurihara and Nishimura 2020). In our department, intraoperative AE is not noted if the bed angle is strictly $30^{\circ}$ or less (Ture et al. 2018). This is probably because at angles below $30^{\circ}$, the venous pressure that flows out and the air pressure that is inhaled are balanced.

The advantages of the semi-sitting position include the lack of cerebellar swelling during the opening of the dura, lower intraoperative blood loss, better control of cerebrospinal fluid/bleeding in the operative field, and easier anatomical orientation. The cerebellum descends as a result of its own weight, and approaches from the top of the cerebellum are easy. This positioning is particularly effective for approaching dorsal midbrain lesions and is useful in the dorsolateral transcondylar approach (Bertalanffy and Seeger 1991; Bertalanffy et al. 2010).

MVD for glossopharyngeal neuralgia in the semi-sitting position is frequently performed in Europe, with only 19 consecutive patients' recent reports showing that preoperative 3D MRI was effective for identifying neurovascular compression before surgery (Gaul et al. 2011). In a report from the United States regarding glossopharyngeal neuralgia MVD in the semi-sitting position, Jannetta (1980) has previously reported it as a modified sitting position. On the other hand, in Japan, there was only one report about the effectiveness of MVD for glossopharyngeal neuralgia in the sitting position (Yonekawa 2010a); to our knowledge, this paper is the first to report the use of the semi-sitting.

In addition to MVD for trigeminal neuralgia, with its approach from the top of the cerebellum, the semi-sitting position in MVD for HFS and glossopharyngeal neuralgia involving the proximal VA trunks can be effective, espe- 

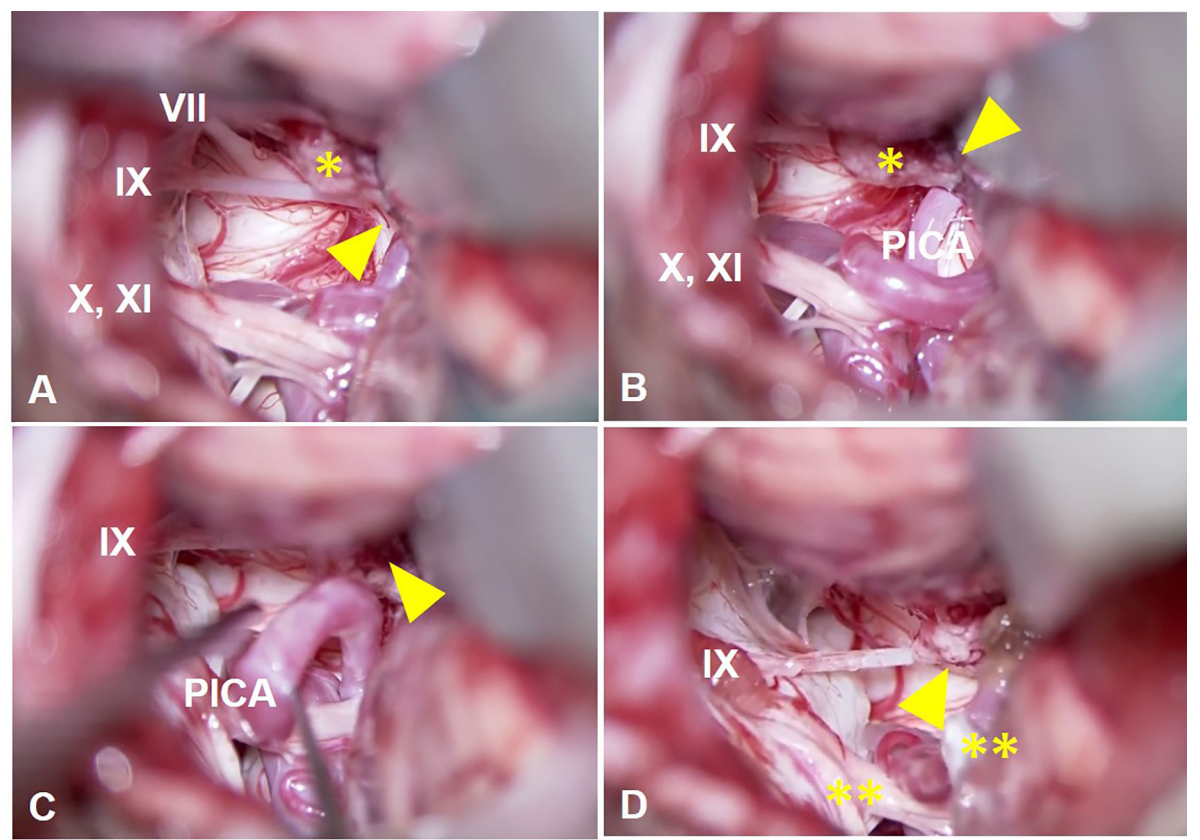

Fig. 4. Surgical procedure performed in Case 2.

A. Intraoperative images of the left microvascular decompression (MVD) for glossopharyngeal neuralgia. Facial (VII) nerve and glossopharyngeal (IX) nerve root entry zone (REZ). The arrowhead indicates glossopharyngeal (IX) nerve REZ. B. Posterior inferior cerebellar artery (PICA) is close to the glossopharyngeal (IX) nerve REZ. The arrowhead indicates the REZ. C. PICA loop was pulled out to the caudal side from the glossopharyngeal (IX) REZ. The arrowhead indicates the REZ. D. Decompression of the PICA loop to the cerebellar hemisphere with Teflon tape. Glossopharyngeal (IX) REZ is completely decompressed. The arrowhead indicates the REZ.

VII, facial nerve; IX, glossopharyngeal nerve; X, vagus nerve; XI, spinal accessory nerve; PICA, posterior inferior cerebellar artery.

*Flocculus, **Teflon tape.

cially in young patients, to approach the brainstem from below and observe the REZ.

The biggest concern with surgery in the semi-sitting position is intraoperative AE. It is necessary to be careful of the veins that run between the fascia muscles; concomitant and emissary veins of the occipital artery; epidural veins, including those encountered during the opening of the dura; and the diploic veins of the craniotomy (Yonekawa 2010b). To promptly detect minute AEs, continuous intraoperative precordial Doppler monitoring and close observation of the end-tidal $\mathrm{CO}_{2}$ are essential (Jadik et al. 2009; Saladino et al. 2017; Ture et al. 2018). To detect and prevent $\mathrm{AE}$ at an early stage, close cooperation with anesthesiologists, effective hemostasis, and monitoring at each stage during the operation are important.

In Japan, there are no recent reports on the use of sitting position in neurosurgery (Yamashima et al. 1999; Higashino et al. 2012). This is because of the increased risk of AE complications when this position is used. Thus, anesthesiology departments do not prefer to use this position. However, in recent years, the usefulness and safety of the semi-sitting position has been reported, mainly from studies in Europe (Saladino et al. 2017; Ture et al. 2018). Thus, the effectiveness of the semi-sitting position has been reviewed in Japan, and a few reports using this position have been published (Ichimura et al. 2018a, b).
If $\mathrm{AE}$ is suspected due to intraoperative precordial Doppler sound and waveform changes, the anesthesiologist first confirms changes in end-tidal $\mathrm{CO}_{2}$ and then administers pure oxygen. At the same time, the anesthesiologist aspirates air from the central venous catheter. Subsequently, the anesthesiologist performs manual compression of the jugular vein and the neurosurgeon confirms the bleeding site that is the site of air entry. Once the bleeding site is confirmed, hemostasis is attempted using bone wax or gel foam. If the neurosurgeon does not locate the bleeding source, the angle of the bed is reduced and the surgical field is re-examined. In addition, if end-tidal $\mathrm{CO}_{2}$ does not improve and significant changes in vital signs occur, it is necessary to stop the surgery.

There may be concerns about choosing the semi-sitting position instead of the traditional park bench position when performing MVD for glossopharyngeal neuralgia. However, the semi-sitting position offers excellent access to the lower cranial nerves, facilitating decompression of the offending vessels inferior to the REZ of the glossopharyngeal nerve and minimizing the risk of intraoperative AE. The semi-sitting position may also be beneficial in avoiding lower cranial nerve complications (Rey-Dios and CohenGadol 2013; Xia et al. 2018).

In conclusion, use of the semi-sitting position in MVD for glossopharyngeal neuralgia provides an excellent surgi- 
cal view of the brainstem. Furthermore, a small craniotomy is sufficient for the semi-sitting position, thus also making the surgery minimally invasive. However, intraoperative monitoring and management are important to avoid AE.

\section{Conflict of Interest}

The authors declare no conflict of interest.

\section{References}

Bertalanffy, H., Bozinov, O., Sürücü, O., Sure, U., Benes, L., Kappus, C. \& Krayenbuhl, N. (2010) Dorsolateral Approach To The Craniocervical Junction. In Cranial, Craniofacial and Skull Base Surgery, edited by Cappabianca, P., Ioaconetta, G. \& Califano, L. Springer, Milano, pp. 175-196.

Bertalanffy, H. \& Seeger, W. (1991) The dorsolateral, suboccipital, transcondylar approach to the lower clivus and anterior portion of the craniocervical junction. Neurosurgery, 29, 815-821.

Gaul, C., Hastreiter, P., Duncker, A. \& Naraghi, R. (2011) Diagnosis and neurosurgical treatment of glossopharyngeal neuralgia: clinical findings and 3-D visualization of neurovascular compression in 19 consecutive patients. J. Headache Pain, 12, 527-534.

Higashino, Y., Kodera, T., Yamauchi, T., Tsunetoshi, K., Hosoda, T., Arishima, H., Kitai, R., Arai, Y., Takeuchi, H. \& Kikuta, K. (2012) Microsurgical resection of an arteriovenous malformation of the pineal region through the infratentorial supracerebellar approach with the patient in a sitting position. Jpn. J. Neurosurg., 21, 244-248.

Ichimura, S., Bertalanffy, H., Nakaya, M., Mochizuki, Y., Fukaya, R., Moriwaki, G., Fukuchi, M. \& Fujii, K. (2018a) Improvement of respiratory depression in a patient with primary medullary hemorrhage following removal of hematoma in the half-sitting position. J. Neurol. Surg. A Cent. Eur. Neurosurg., 79, 186-190.

Ichimura, S., Bertalanffy, H., Nakaya, M., Mochizuki, Y., Moriwaki, G., Sakamoto, R., Fukuchi, M. \& Fujii, K. (2018b) Surgical treatment for primary brainstem hemorrhage to improve postoerative functional outcomes. World Neurosurg., 120, e1289-e1294.

Jadik, S., Wissing, H., Friedrich, K., Beck, J., Seifert, V. \& Raabe, A. (2009) A standardized protocol for the prevention of clinically relevant venous air embolism during neurosurgical interventions in the semisitting position. Neurosurgery, 64, 533-538; discussion 538-539.

Jannetta, P.J. (1980) Neurovascular compression in cranial nerve and systemic disease. Ann. Surg., 192, 518-525.

Kawashima, M., Matsushima, T., Inoue, T., Mineta, T., Masuoka, J. \& Hirakawa, N. (2010) Microvascular decompression for glossopharyngeal neuralgia through the transcondylar fossa (supracondylar transjugular tubercle) approach. Neurosur- gery, 66, 275-280; discussion 280.

Kurihara, M. \& Nishimura, S. (2020) Estimation of the head elevation angle that causes clinically important venous air embolism in a semi-sitting position for neurosurgery: a retrospective observational study. Fukushima J. Med. Sci., 66, 67-72.

Laha, R.K. \& Jannetta, P.J. (1977) Glossopharyngeal neuralgia. J. Neurosurg., 47, 316-320.

Malis, L.I. (1998) Acoustic Neuroma, Elsevier, Amsterdam.

Revuelta-Gutierrez, R., Morales-Martinez, A.H., Mejias-Soto, C., Martinez-Anda, J.J. \& Ortega-Porcayo, L.A. (2016) Microvascular decompression for glossopharyngeal neuralgia through a microasterional approach: a case series. Surg. Neurol. Int., 7, 51

Rey-Dios, R. \& Cohen-Gadol, A.A. (2013) Current neurosurgical management of glossopharyngeal neuralgia and technical nuances for microvascular decompression surgery. Neurosurg. Focus, 34, E8.

Saladino, A., Lamperti, M., Mangraviti, A., Legnani, F.G., Prada, F.U., Casali, C., Caputi, L., Borrelli, P. \& DiMeco, F. (2017) The semisitting position: analysis of the risks and surgical outcomes in a contemporary series of 425 adult patients undergoing cranial surgery. J. Neurosurg., 127, 867-876.

Samii, M. \& Matthies, C. (1996) Hearing Preservation In Acoustic Tumor Surgery. In Current Techniques in Neurosurgery, edited by Salcman, M., Current Medicine, Philadelphia, PA, pp. 93-108.

Samii, M. \& Matthies, C. (1997) Management of 1000 vestibular schwannomas (acoustic neuromas): surgical management and results with an emphasis on complications and how to avoid them. Neurosurgery, 40, 11-21; discussion 21-23.

Tanrikulu, L., Hastreiter, P., Dorfler, A., Buchfelder, M. \& Naraghi, R. (2015) Classification of neurovascular compression in glossopharyngeal neuralgia: three-dimensional visualization of the glossopharyngeal nerve. Surg. Neurol. Int., 6, 189.

Ture, H., Harput, M.V., Bekiroglu, N., Keskin, O., Koner, O. \& Ture, U. (2018) Effect of the degree of head elevation on the incidence and severity of venous air embolism in cranial neurosurgical procedures with patients in the semisitting position. J. Neurosurg., 128, 1560-1569.

Yamashima, T., Yamashita, S. \& Kanno, T. (1999) Surgical set up for sitting/semisitting position. Jpn. J. Neurosurg., 8, 262.

Yonekawa, Y. (2010a) Operative neurosurgery: personal view and historical backgrounds (7) reappraisal of approaches. No Shinkei Geka, 38, 1031-1045.

Yonekawa, Y. (2010b) Operative neurosurgery: personal view and historical backgrounds. 6. Positioning, instruments. No Shinkei Geka, 38, 381-396.

Xia, L., Li, Y.S., Liu, M.X., Zhong, J., Dou, N.N., Li, B. \& Li, S.T. (2018) Microvascular decompression for glossopharyngeal neuralgia: a retrospective analysis of 228 cases. Acta Neurochir. (Wien), 160, 117-123. 